\title{
Multiple stellar populations in the Galactic globular cluster NGC $6752^{1}$
}

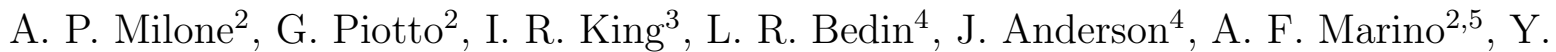 \\ Momany $^{6}$, L. Malavolta ${ }^{2,7}$, and S. Villanova ${ }^{8}$
}

\begin{abstract}
We have carried out high-precision photometry on a large number of archival HST images of the Galactic globular cluster NGC 6752, to search for signs of multiple stellar populations. We find a broadened main sequence, and demonstrate that this broadening cannot be attributed either to binaries or to photometric errors. There is also some indication of a main-sequence split. No significant spread could be found along the subgiant branch, however. Ground-based photometry reveals that in the $U$ vs. $(U-B)$ color-magnitude diagram the red-giant branch exhibits a clear color spread, which we have been able to correlate with variations in $\mathrm{Na}$ and $\mathrm{O}$ abundances. In particular the Na-rich, O-poor stars identified by Carretta et al. (2007) define a sequence on the red side of the red-giant branch, while Na-poor, O-rich stars populate a bluer, more dispersed portion of the red-giant branch.
\end{abstract}

Subject headings: globular clusters: individual (NGC 6752) — HertzsprungRussell diagram

\footnotetext{
${ }^{2}$ Dipartimento di Astronomia, Università di Padova, Vic. Osservatorio 3, I-35122, PD, Italy; [antonino.milone,giampaolo.piotto,anna.marino,luca.malavolta]@unipd.it

${ }^{3}$ Department of Astronomy, University of Washington, Seattle, WA 98195-1580, USA; king@astro.washington.edu

${ }^{4}$ Space Telescope Science Institute, 3800 San Martin Drive, Baltimore, MD 21218; [jayander,bedin]@stsci.edu

${ }^{5}$ P. Universidad Católica de Chile, Departamento de Astronomía y Astrofísica, Casilla 306, Santiago 22, Chile; fmarino@astro.puc.cl

${ }^{6}$ Osservatorio Astronomico di Padova, Vicolo dell'Osservatorio 5, 35122 Padova, Italy; yazan.momany@oapd.inaf.it

${ }^{7}$ Visiting undergraduate Student at STScI under the 2009 Space Astronomy Summer Program, http://www.stsci.edu/institute/sd/students

${ }^{8}$ Departamento de Astronomia, Universidad de Concepcion, Casilla 160-C, Concepcion, Chile; svillanova@astro-udec.cl
} 


\section{Introduction}

For several decades astronomers have believed that globular clusters (GCs) were made up of stars that were all born at the same time and out of the same material, and that as such, they offered the best approximation to a simple stellar population (see, e.g., Renzini \& Buzzoni 1986). The abundance anomalies observed among GC stars since the early seventies (see Gratton et al. 2004 for a recent review) have challenged this scenario, but it was only the recent discovery of clear photometric evidence of multiple stellar populations among unevolved stars that destroyed once and for all this traditional scenario, moving the study of GCs in a new direction.

The most striking case is $\omega$ Centauri, which exhibits large star-to-star iron variations (see Villanova et al. 2007 and references therein) with evidence of multiple red-giant branches (RGBs, seen earlier by Lee et al. 1999 and Pancino et al. 2000), multiple subgiant branches (SGBs), and at least three distinct main sequences (MSs, Bedin et al. 2004). Furthermore, Piotto et al. (2005) showed that the red MS is more metal poor than the blue MS, which may imply that the latter has a strong He enhancement (as suggested by Norris 2004).

High-accuracy photometry from Hubble Space Telescope (HST) images has allowed us to discover that NGC 2808 also hosts multiple distinct MSs (in this case three; see Piotto et al. 2007), possibly associated with three stellar populations with different He content and with the multi-modal horizontal branch (HB, D'Antona et al. 2005). In addition, we have detected a split SGB in NGC 1851 (Milone et al. 2008), M22 (NGC 6656; Piotto 2009, Marino et al. 2009), NGC 6388 (Moretti et al. 2009), and many other GCs, both in the Milky Way (Piotto 2009) and in the Magellanic Clouds (Mackey et al. 2008, Milone et al. 2009). More recently, Anderson et al. (2009) have found that the MS of 47 Tuc (NGC 104) is also spread much more than can be expected from photometric errors, while the SGB shows at least two distinct components.

The nearby globular cluster NGC $6752\left(d=4.0 \mathrm{kpc}, M_{V}=-7.73\right.$, Harris 1996) is another very promising candidate to examine for multiple populations. There is evidence of strong abundance anomalies (Carretta et al. 2007), and it has an extended blue HB, similar to the HBs of NGC 2808, $\omega$ Cen, and M54 (NGC 6715), all clusters in which mixed stellar populations have recently been found.

In this paper we will use both $H S T$ and ground-based photometry to study the colormagnitude diagram (CMD) of NGC 6752 for signs of multiple stellar populations. HST data

\footnotetext{
${ }^{1}$ Based on observations with the NASA/ESA Hubble Space Telescope, obtained at the Space Telescope Science Institute, which is operated by AURA, Inc., under NASA contract NAS 5-26555.
} 
will allow us to carefully analyze the MS and the SGB in the central field, while groundbased data will be used to examine the RGB. One careful photometric study of the MS of NGC 6752 already exists: Rubenstein \& Bailyn (1997, RB97) used HST's WFPC2 camera to study the MS of the cluster, and concluded that there was a broadening toward the red side that could be explained by the presence of a considerable fraction of binaries near the center of the cluster. We will reconcile our results with those of RB97, by re-reducing their images with the techniques of today and by introducing new measurements of images from HST's Advanced Camera for Surveys (ACS), as well as ground-based images with a larger field. 


\section{Observations and data reduction}

In order to search for signs of multiple stellar populations in the crowded core of NGC 6752, we retrieved a large and varied set of images from the $H S T$ archive. These images are listed in Table 1, while Fig. 1 shows their footprints. One of the problems of identifying multiple populations, or else spreads in cluster sequences, is the fact that photometric errors can introduce similar signatures. Anderson et al. (2009) have shown that a good way to distinguish real broadening from mere photometric error is to analyze independent data sets, and see if they exhibit the same features. Although the archival material available for NGC 6752 it is not as extensive as the set of images that Anderson et al. had for 47 Tuc, we were able to make use of several different HST programs whose ACS images used both the WFC and the HRC channels, and were at different pointings and roll angles and used different filter pass-bands.

From the archival HST images of NGC 6752 in various passbands, we were able to make five completely independent CMDs. Four of our ACS CMDs come from WFC images, and a fifth from HRC images:

1) $m_{\mathrm{F} 555 \mathrm{~W}}$ and $m_{\mathrm{F} 814 \mathrm{~W}}$ from GO-10121;

2) $m_{\mathrm{F} 606 \mathrm{~W}}$ and $m_{\mathrm{F} 814 \mathrm{~W}}$ from GO-10775;

3) $m_{\mathrm{F} 475 \mathrm{~W}}$ from GO-9899 and $m_{\mathrm{F} 606 \mathrm{~W}}$ from GO-10459;

4) $m_{\mathrm{F} 606 \mathrm{~W}}$ and $m_{\mathrm{F} 814 \mathrm{~W}}$ from GO-9453;

5) $m_{\mathrm{F} 435 \mathrm{~W}}$ and $m_{\mathrm{F} 555 \mathrm{~W}}$ from GO-10335 (both taken with the HRC).

Data sets 1-4 allowed us to study the MS (Sect. 3), while data sets 1, 2, 4, and 5 were used to study the structure of the SGB (Sect. 4).

We also used, for comparison purposes, the WFPC2 images of Rubenstein \& Bailyn (1997), from their GO-5318. The results of our remeasurement of those images will be presented in the following section.

The ACS/WFC images were reduced by using the procedure described in Anderson et al. (2008), which allowed us to analyze all the exposures of each data set simultaneously to generate a single star list. Stars are measured independently in each image by using a spatially varying $9 \times 10$ array of empirical "library PSFs" from Anderson \& King (2006), plus a spatially constant perturbation for each exposure, to allow for variations in the telescope focus. The software is able to detect almost every star that would be found by eye. It was designed to work well in both crowded and uncrowded fields, and takes advantage of the many independent dithered pointings and the knowledge of the PSF, to avoid including artifacts in the list. The photometry was put into the ACS Vega-mag system following recipes in Bedin et al. (2005) and using the zero points given in Sirianni et al. (2005). Unfortunately, the hybrid PSF model above is not able to account for all of the effects of telescope breathing, 
which can introduce a small spatial dependence of the shape of the PSF, which is not compensated for in our PSF model and can cause small systematic photometric errors that depend on position on the detector. The typical variation is small (about $1 \%$ in the fraction of light in the core). To account for the color differences that these variations produce, we used the following procedure: first we drew a main-sequence ridge line (MSRL), by putting a spline through the median colors found in successive short intervals of magnitude, and we iterated this step with a sigma clipping; then we examined the color residuals relative to this sequence, as a function of location on the detector. These color variations can come from either differential reddening or from PSF variation, but we make empirical star-by-star corrections, regardless of the cause. We compute for each star its color residual from the MSRL, and then correct the star's color by the difference between its color residual and the mean of those of its best-measured neighbors (50 to 100 of them, according to the detector and the star density). These corrections are typically smaller than $0.005 \mathrm{mag}$, and never exceed $0.013 \mathrm{mag}$, but including them makes a significant improvement in our photometric results.

The measurement of stellar fluxes and positions in each ACS/HRC image was performed by using the publicly available measuring routine, library PSFs, and the distortion correction described in Anderson \& King (2004). We corrected the zero points of color in the same way as for the WFC photometry.

As for the PC images from GO-5318, we used the same procedures as for other WFPC2 images in Bedin et al. (2001).

Finally, since our focus here is on high-quality photometry, we included in the analysis only relatively isolated, unsaturated stars with good values of the PSF-fit quality index and small rms errors in photometry and in astrometry. A detailed description of the selection procedures is given in Milone et al. (2009).

In addition, we used artificial-stars (AS) for several purposes: to determine the completeness level of our sample, to estimate the internal photometric errors, and to measure the fraction of chance-superposition binaries. The AS experiments followed the recipes of Anderson et al. (2008), while the procedure used to determine a position-dependent completeness is described in Milone et al. (2009).

The HST fields are too small to include a statistically significant number of giant stars, so in order to study the distribution of the stars along the RGB (Sect. 5), we analyzed the photometric catalogs obtained from the Wide Field Imager (WFI) of the ESO/MPI 2.2m telescope, already presented in Momany et al. (2002). The ground-based $U$ and $B$ images of NGC 6752 were taken on July 25-26, 2000. The WFI camera consists of eight $2048 \times 4096$ 


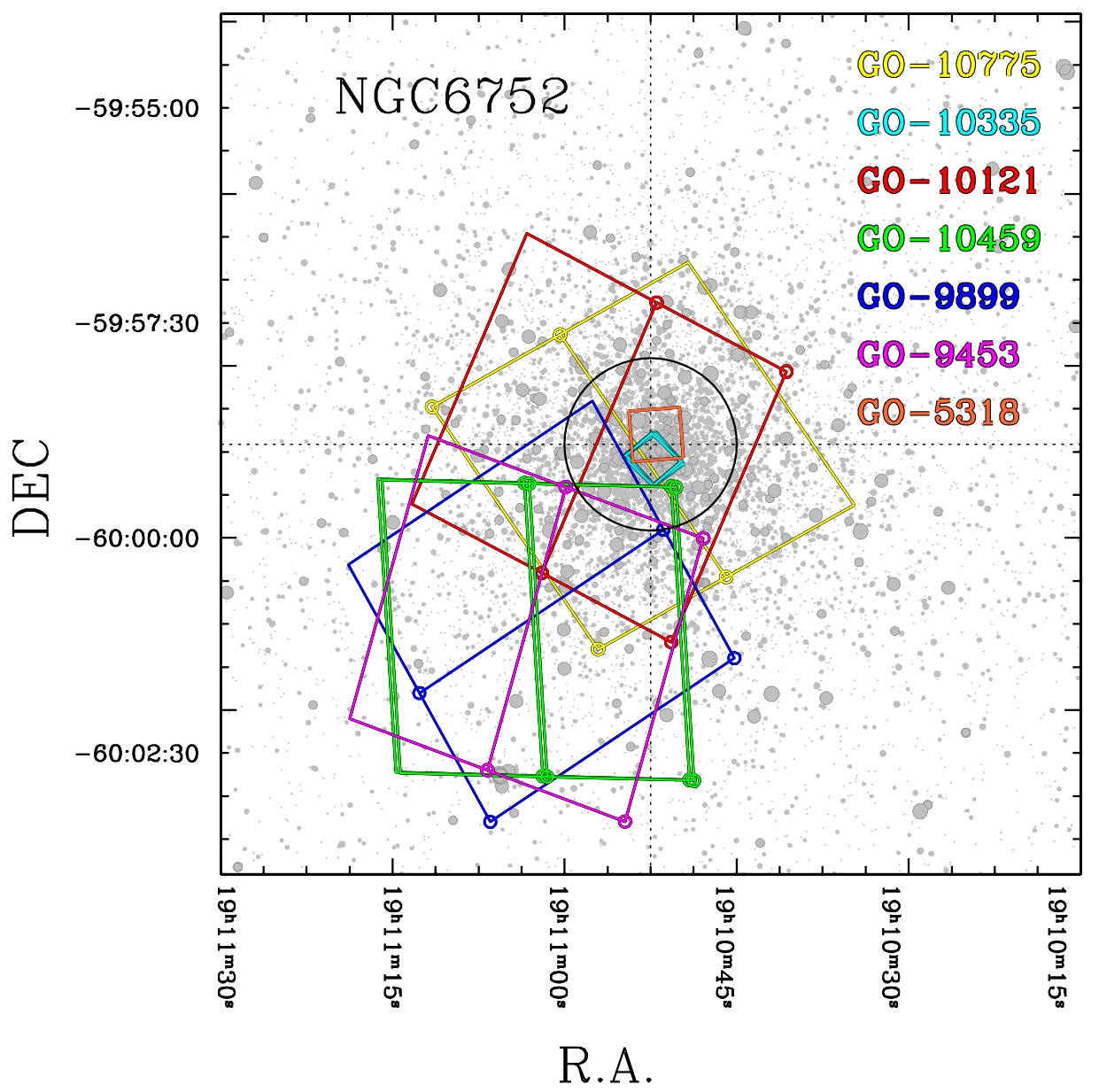

Fig. 1.- Footprints of the HST/ACS data sets used in this work. The small orange box at the center is the footprint of the $\mathrm{PC}$ field of $\mathrm{R} \& \mathrm{~B}$. The small circles mark the corners of WFC chip \#1. 
EEV CCDs, with a total field of view of $34 \times 33$ arcmin. The exposure times (30s and 150s in $U, 5 \mathrm{~s}$ and $10 \mathrm{~s}$ in $B$ ) were chosen in order to sample both the bright RGB and the upper MS stars. Weather conditions were photometric, with good seeing (better than 0.8 arcsec FWHM for all images). Basic reductions of the CCD mosaic were performed using the IRAF package MSCRED (Valdes 1998), while stellar photometry was performed using the DAOPHOT and ALLFRAME programs (Stetson 1994). Finally, instrumental magnitudes were calibrated to the $U B V$ standard system by observing, on each of the eight chips, a field of standard stars from Landolt (1992), during the same nights as the NGC 6752 observations. 


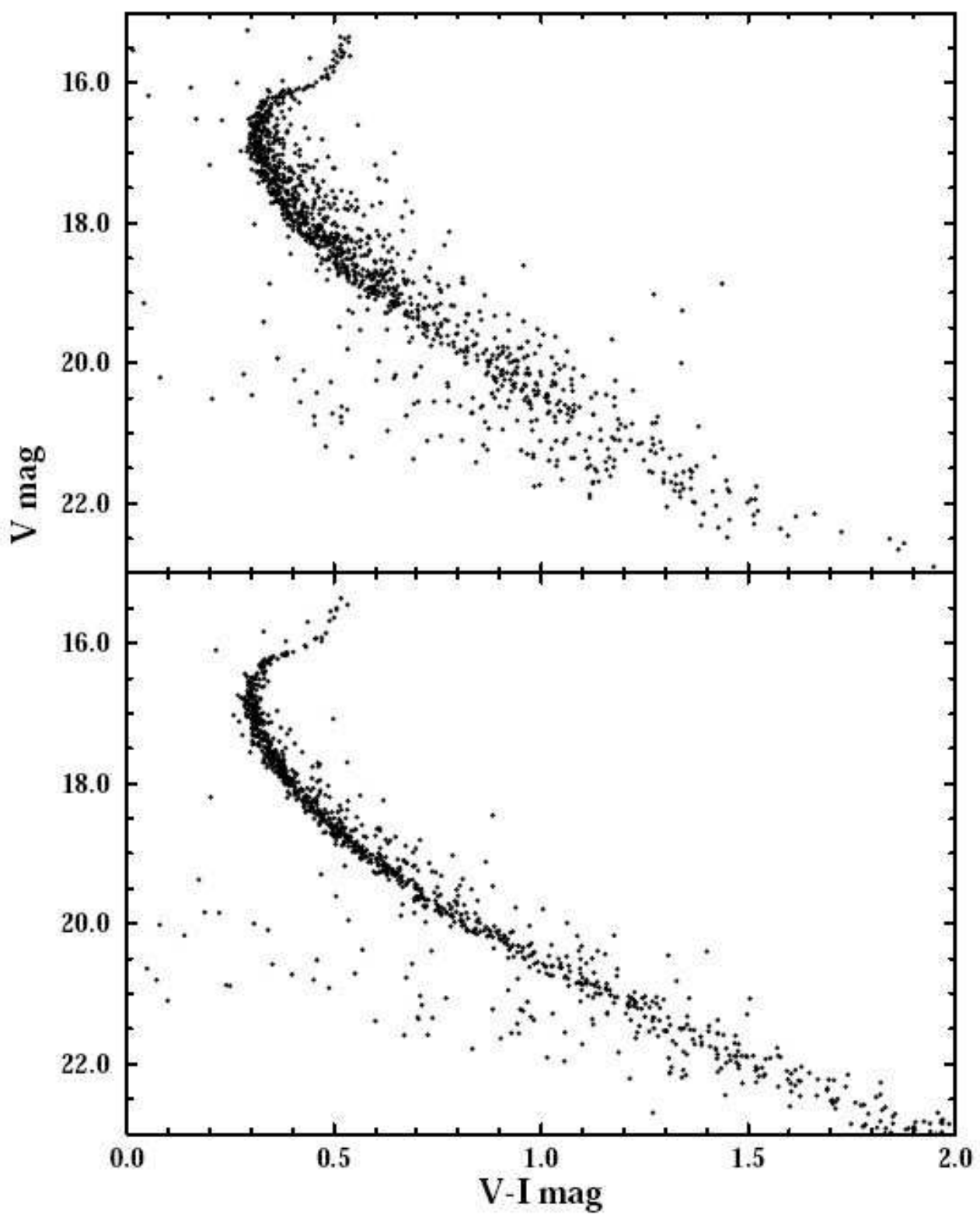

Fig. 2.- Reproduction of Fig. 5 of Rubenstein \& Bailyn (1997). The upper half shows their CMD for the cluster core $(r<11$ arcsec) and the lower half their CMD for the remainder of the PC field. 


\section{The Main Sequence}

Our aim here is to look for a possible broadening of the MS. This could come from an intrinsic spread in the character of the MS stars, but such an appearance might also be the result of binaries, which are seen in many clusters as a spread on the red side of the MS. The organization of this section is therefore as follows: First we take up the question of binaries

and their possible influence on our results, which we show to be small in comparison with the broadening that we observe. Then we present the results from our best data set, from GO-10121, which show the structure of the MS most clearly. Finally, we confirm the MS spread by comparing photometry from other ACS data sets with that from GO-10121.

\subsection{The possible influence of binaries}

In a paper that set a standard of photometric accuracy for its time, RB97 used HST's WFPC2 camera to study the MS of NGC 6752, and concluded that there was a broadening toward the red side that could be explained by the presence of an admixture of at least $15 \%$ binaries near the center of the cluster. We now re-examine their result, first by using the improved photometric techniques that are now available, and then using the better data sets that have been collected more recently.

The data available to RB97, in program GO-5318 (P.I. Bailyn), consisted of somewhat more than 100 PC images in each of the filters F555W and F814W. (Since their observations were made in a rapid-fire repetition, limitations of downlink speed prevented them from recording the WF data.) Their measurements strongly suggested the presence of a quite significant number of MS binaries near the cluster center, and a smaller fraction in the outer part of their field, (as would be expected from the greater central concentration of the binaries, on account of their masses). Their Fig. 5 is reproduced here as our Fig. 2, We have remeasured their images, using the improved photometric techniques of Anderson \& King (1999, 2000, 2003); our resulting CMDs are shown in Fig. 3, left panel. The dashed lines in Fig. 3 show the locus of equal-mass binaries. We now find, from the images that RB97 used a dozen years ago, a strikingly smaller spread than is stated in their paper. The difference is due not to any error on their part, but to the photometric improvement referred to above which allows us better to resolve stellar blends. We see that with our improved measurements the evidence for binaries is almost completely gone. It is of course very unlikely that this

cluster is devoid of binaries; but they are concentrated to the central region, where they represent only a tiny fraction of the cluster population.

The evidence for binaries is even further decreased when we use the photometrically 


\begin{tabular}{cccccl}
\hline \hline INSTR & DATE & N $\times$ EXPTIME & FILTER & PROGRAM & PI \\
\hline WFC & Sep 15 2002 & $1 \times 4 \mathrm{~s}+1 \times 40 \mathrm{~s}$ & F606W & GO-9453 & Brown \\
WFC & Sep 15 2002 & $1 \times 4 \mathrm{~s}+1 \times 46 \mathrm{~s}$ & F814W & GO-9453 & Brown \\
WFC & Jul 18 2004 & $6 \times 340 \mathrm{~s}$ & F475W & GO-9899 & Piotto \\
WFC & Sep 19 2004 & $11 \times 435 \mathrm{~s}+12 \times 80 \mathrm{~s}$ & F555W & GO-10121 & Bailyn \\
WFC & Sep 19 2004 & $12 \times 40 \mathrm{~s}$ & F814W & GO-10121 & Bailyn \\
WFC & Oct 16 2005 & $8 \times 450 \mathrm{~s}$ & F606W & GO-10459 & Biretta \\
WFC & May 24 2006 & $1 \times 2 \mathrm{~s}+4 \times 35 \mathrm{~s}$ & F606W & GO-10775 & Sarajedini \\
WFC & May 24 2006 & $1 \times 2 \mathrm{~s}+4 \times 40 \mathrm{~s}$ & F814W & GO-10775 & Sarajedini \\
HRC & Jun 08 2004 and Jun 05 2006 & $24 \times 35 \mathrm{~s}$ & F435W & GO-10335 & Ford \\
HRC & Jun 08 2004 and Jun 05 2006 & $12 \times 10 \mathrm{~s}$ & F555W & GO-10335 & Ford \\
WFPC2 & Aug 18-19 1994 & $117 \times 26 \mathrm{~s}+26 \times 80 \mathrm{~s}$ & F555W & GO-5318 & Bailyn \\
WFPC2 & Aug 18-19 1994 & $107 \times 50 \mathrm{~s}+27 \times 160 \mathrm{~s}$ & F814W & GO-5318 & Bailyn \\
WFI@2.2m & Jul 25-26 2000 & $4 \times 30 \mathrm{~s}+4 \times 150 \mathrm{~s}$ & $U$ & 065.L-0561 & Piotto \\
WFI@2.2m & Jul 25-26 2000 & $3 \times 5 \mathrm{~s}+4 \times 10 \mathrm{~s}$ & $B$ & 065.L-0561 & Piotto \\
\hline \hline
\end{tabular}

Table 1: Description of the data sets used in this paper.
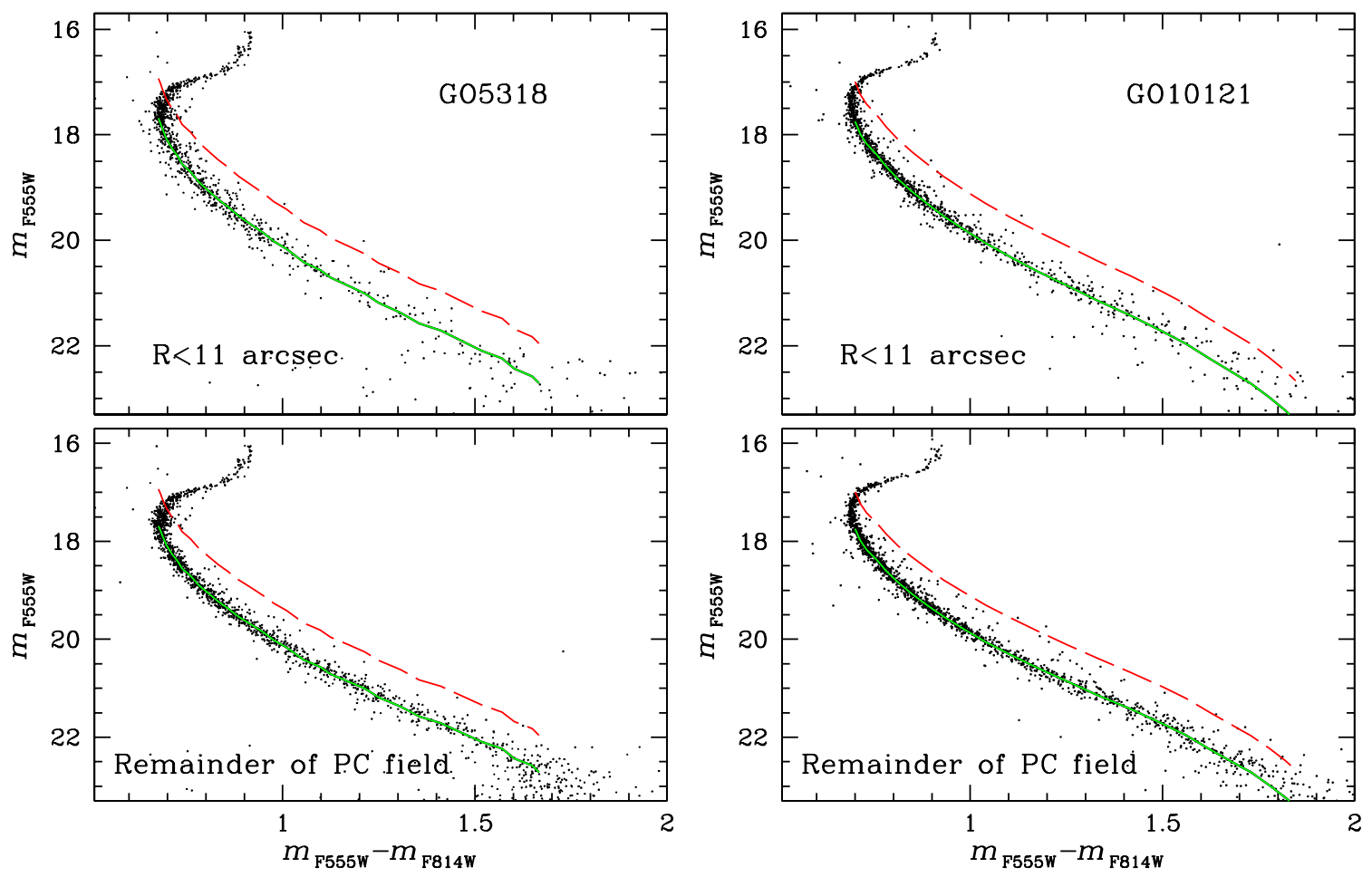

Fig. 3.- Left panels: As in Fig. 2, but showing our CMDs derived from the same images. Right panels: CMDs for the same regions covered by the R\&B CMDs (Fig. 2) and the CMDs in the left panels, but from photometry done on the GO-10121 ACS images. The dashed lines show the locus of equal-mass binaries. 
superior images that ACS provides (Fig. 3, right panels).

In order to put a more quantitative constraint on the binary fraction in NGC 6752, we created five simulated CMDs, assuming binary fractions $F_{\text {bin }}=0.00,0.05,0.10,0.30,1.00$, respectively. The simulations used random choices of artificial stars, as an adaptation of the procedures of Sollima et al. (2007). What we did was to make random choices from the Kroupa IMF (2002), a star at a time for the MS, and also pairs to simulate binaries. Using a mass-luminosity relation (whose exact choice is unimportant) and an assumed distance modulus, we calculated an $m_{\mathrm{F} 814 \mathrm{~W}}$ for each star, and assigned the star the corresponding MS color; finally for each binary we summed the flux in each band and derived the resulting magnitude and color.

In Fig. 4 we compare each of the simulated CMDs with our observed one, using the sample with the best photometry, namely the part of the GO-10121 ACS field that has $r>1$ arcmin. A cursory comparison between observed and simulated CMDs suggests that the binary fraction is small, but we make a more accurate measure of it by using a procedure that is illustrated in Figure 5. The upper half of the figure shows two regions of the CMD, A (upper left) and B (upper right). Region A is drawn so as to contain all the stars that we can consider to be cluster members. The green continuous line is the main-sequence ridge line, drawn by the method that we described in Sect. 2, To include stars moved to the blue by measuring error, we extend region $\mathrm{A}$ as far as the green dashed line, which is displaced to the blue from the MSRL by three times the rms measuring error of the colors (calculated as a function of $m_{\mathrm{F} 814 \mathrm{~W}}$ ). The red dotted line is the locus of binaries whose components have equal mass; we set the other limit of region A by drawing the red dot-dash line, displaced this time to the red from the dotted line by three times the rms color error.

The upper-right panel of Fig. 5 shows Region B, which is chosen in such a way that we can be reasonably sure that all stars in it are binaries. It commences at the locus of binaries with ratio of mass of the secondary to that of the primary, $q=m_{2} / m_{1}=0.5$, marked by the continuous red line, which coincidentally falls three color-error sigmas to the red of the MSRL, and it ends at the dashed red line, which is again the $3 \sigma$ redward bound of equal-mass binaries, just as in the upper-left panel.

The lower half shows how the stars fall with respect to these two regions. On the left are the observed stars; there are 9048 of them in region A, but only 185 of these fall in region B. On the right are artificial stars, which we chose with random positions and with random magnitudes but MSRL colors. Of the 9126 ASs, 112 fell in region B. These stars are too far from the MSRL to be explained by measuring error; the real explanation must be that in those 112 cases two stars fell at positions so close together that a pair of stars has blended into a single image, which would simulate a binary. If we adjust for the sample size (9048 


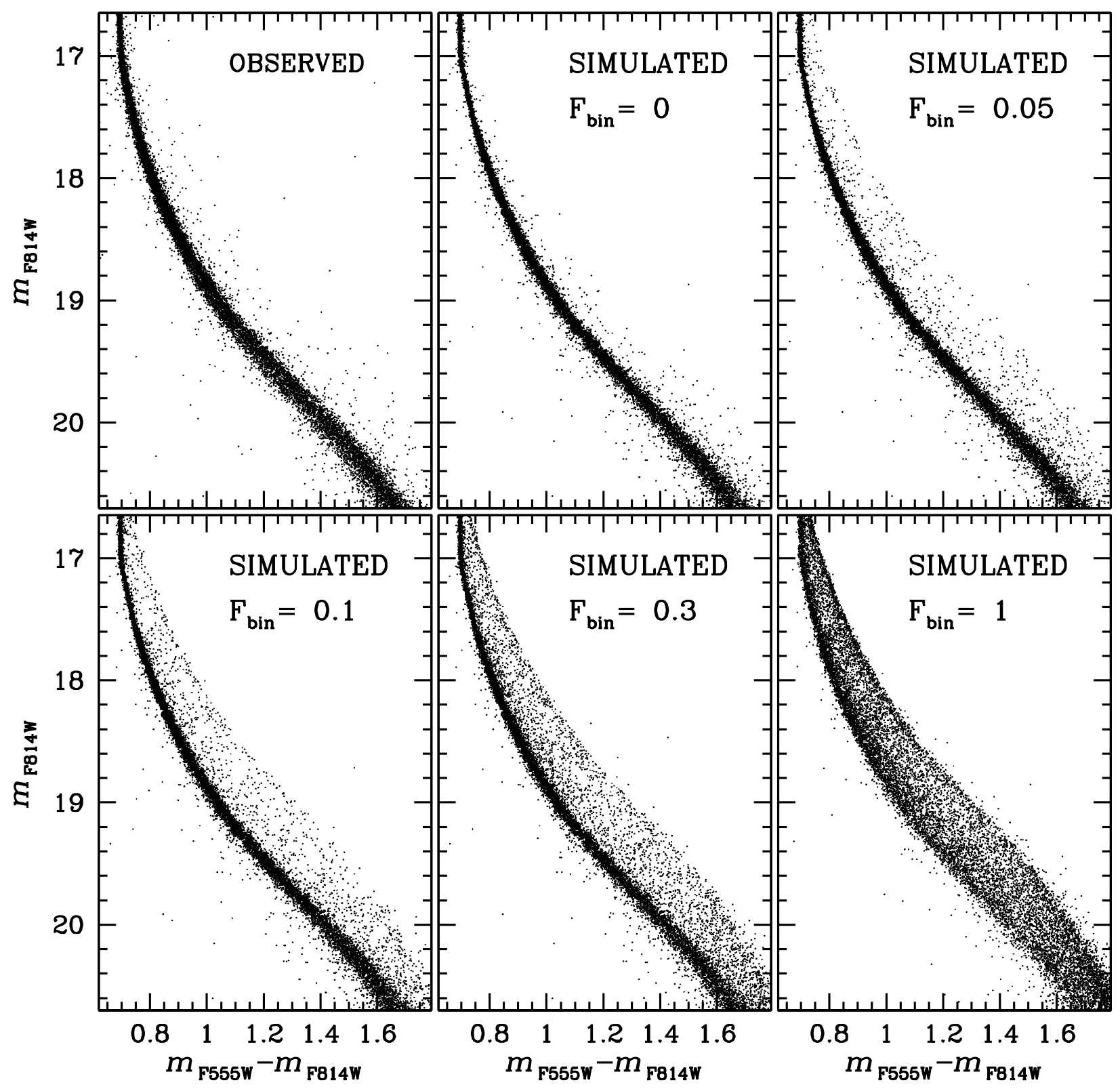

Fig. 4.- Observed and simulated CMDs of NGC 6752. 
instead of 9126), then 111 apparent binaries among the real stars are likely to be explainable as blends, leaving only 74 binaries.

Finally, before we deduce a binary fraction, one more correction is needed: for field stars. To estimate the number of these, we used a program devised by Girardi et al. (2005), which uses a Galactic model to predict star numbers in any given direction. For a field the size of ours in the direction of NGC 6752, the program predicts 19 stars in region A, 14 of which fall within region $\mathrm{B}$. We are thus left with an estimate of $74-14=60$ binaries among 9048 $-19=9029$ total stars, or $0.7 \%$. A similar procedure, applied to the central PC field of the original RB97 images, found a binary fraction of $2.6 \%$.

\subsection{The intrinsic broadening of the main sequence}

Satisfied that we are not being led astray by a serious admixture of binaries, we now take up the question of an intrinsic breadth in the main sequence. As might be expected, our best source of information comes from our best data set, that from GO-10121. Figure 6 shows our $m_{\mathrm{F} 814 \mathrm{~W}}$ vs. $m_{\mathrm{F} 555 \mathrm{~W}}-m_{\mathrm{F} 814 \mathrm{~W}} \mathrm{CMD}$ from this data set. We have applied the corrections for spatial variations of the zero point of colors, as described in Section 2, and have excluded stars less than 1 arcmin from the cluster center, so as to avoid the most crowded regions. (Note that this is a post-core-collapse cluster.)

A visual inspection of this CMD immediately suggests that NGC 6752 has a broad MS. The Hess diagram in the inset reinforces this impression, and even suggests that the cluster could have a second (but less populated) MS, on the blue side of the main MS, in close analogy with the multiple MSs that have been observed in $\omega$ Cen and in NGC 2808. We will not pursue this question further, but hope that future observations will clarify it.

We also note at this point an additional piece of evidence that the broadening that we see is unlikely to be due to binaries. In fact, we already showed that the fraction of binaries with mass ratio $q>0.5$ is smaller than $3 \%$ of the cluster population, even in the core. In order to reproduce the broadening of the MS shown in Fig. 4 with binaries, we would have to make the outlandish assumption that two thirds of the stars in NGC 6752 are in binary systems with mass ratios $q<0.2$.)

Putting aside the question of whether the main sequence is split, and having excluded the possible contribution of binaries, we now return to the basic question of whether it is broader than would be expected from photometric measuring error alone. As was shown in the study by Anderson et al. (2009) of MS broadening in 47 Tuc, a very effective way of testing for a true broadening is to divide the images of the same field of stars into two 


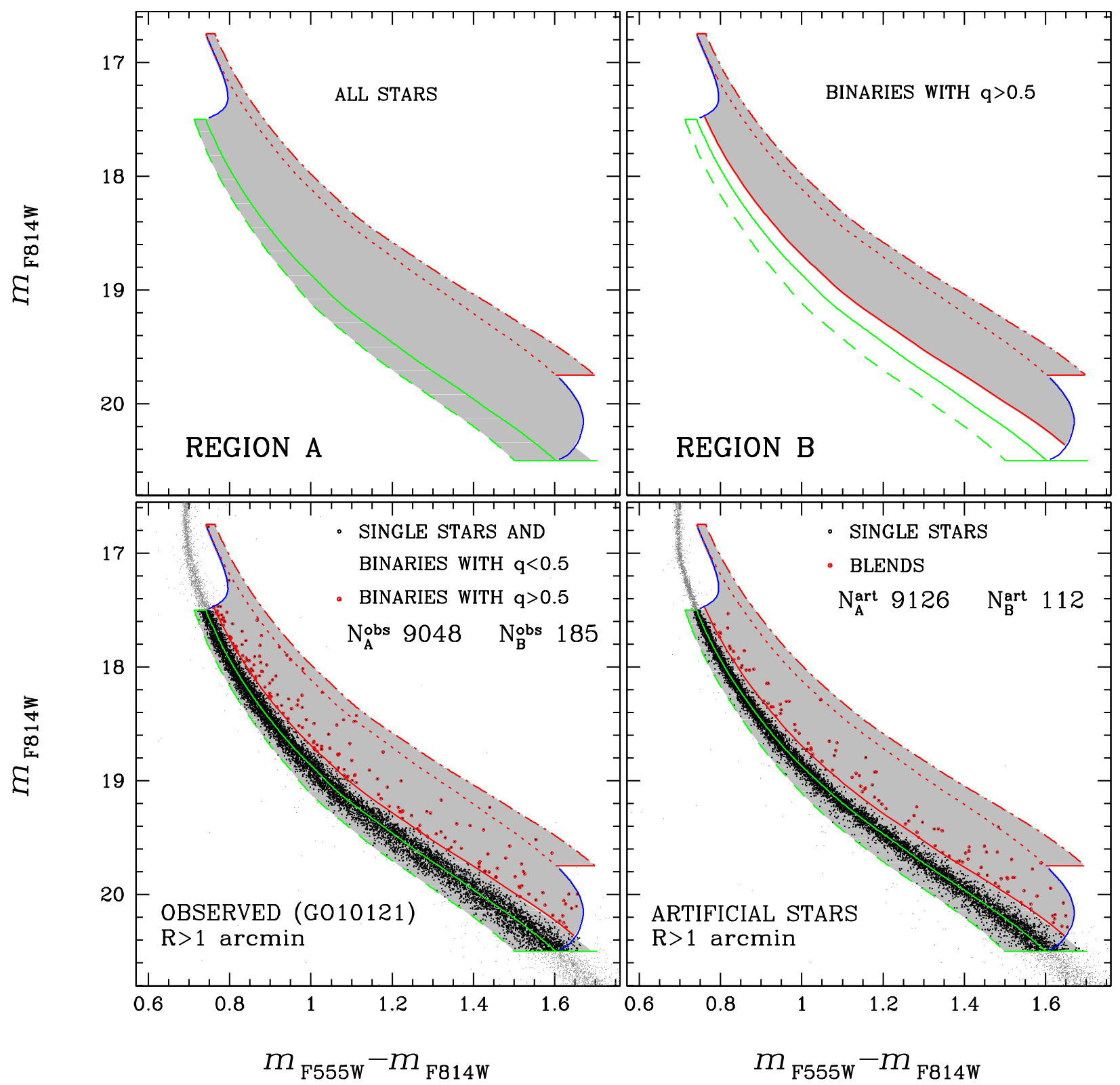

Fig. 5.- The upper half of the figure shows the regions A and B that are described in the text, delineating respectively the region of all cluster stars and the sub-region of binaries with mass ratio greater than 0.5 . In the lower half of the figure the stars are shown, with somewhat larger symbols for the stars in the binary region. At left are the observed stars, and at right the artificial stars described in the text. 


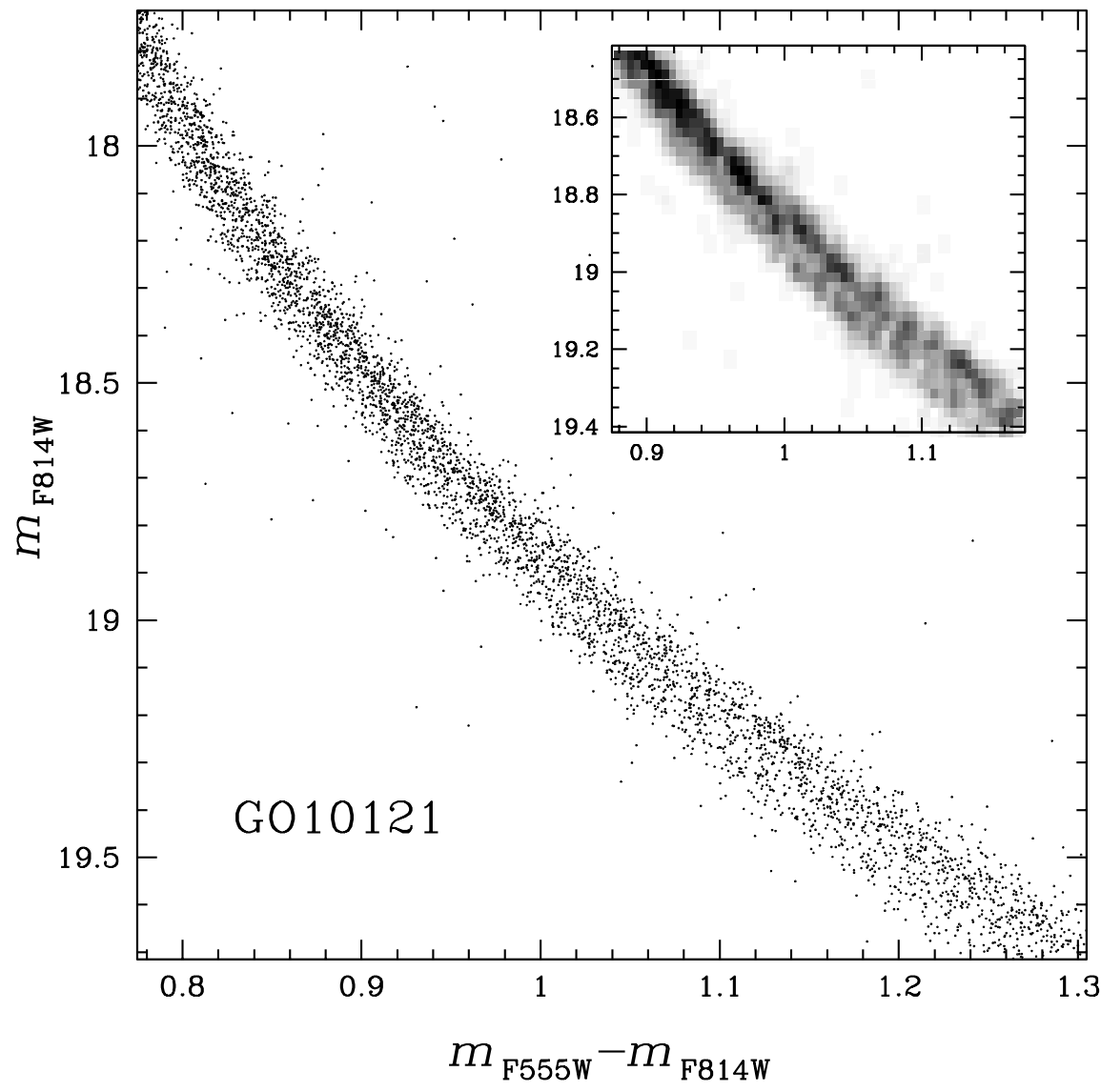

Fig. 6. - CMD of NGC 6752 from GO-10121 ACS/WFC data. The inset shows a Hess diagram for the MS region with $18.4<m_{\mathrm{F} 814 \mathrm{~W}}<19.4$.

independent sets. If the broadening is intrinsic, the stars that have redder (or bluer) colors in the results from one half of the images will have redder (or bluer) colors in the results from the other half. But if the broadening is due only to measuring error, a star that is redder in the first half will have an equal chance of being redder or bluer in the second half, and the bluer stars of the first half are equally likely to be red or blue in the second half. We have therefore drawn a median line in panel (a), and used red and blue colors for the stars on either side of the line, and in panel (b) we have kept for each star the same color that it had in panel (a).

It is quite evident that the colors of stars are maintained very well from panel (a) to panel (b); photometric error has led to only a small mismatch. To emphasize this fact, we show in panel (c) the correlation between the colors of each star in the two halves of the data. Because the measuring errors increase at fainter magnitudes it is clearer to show separate plots for four successive intervals of magnitude. If a star was measured as redder than the 
MSRL in the first half of the data, then in the great majority of cases it is also measured as redder in the second half of the data. This is the mark of a true spread in color.

For further emphasis we show in panel (d) the color distribution of the straightened MS as measured from the whole set of images. The measuring errors of these colors are of course smaller than those of either half of the data set, by a factor of $\sqrt{2}$, and now there is even an intriguing hint again of the split that we suspected in Fig. 6.

Finally, in panel (e) we show the distribution of half the difference between the colors measured separately from each half of the images, which is a statistical estimator of the errors of the colors in panel (d). In these last two panels we have written into each magnitude interval an estimate of the sigma of the spread, as deduced from its interquartile separation.
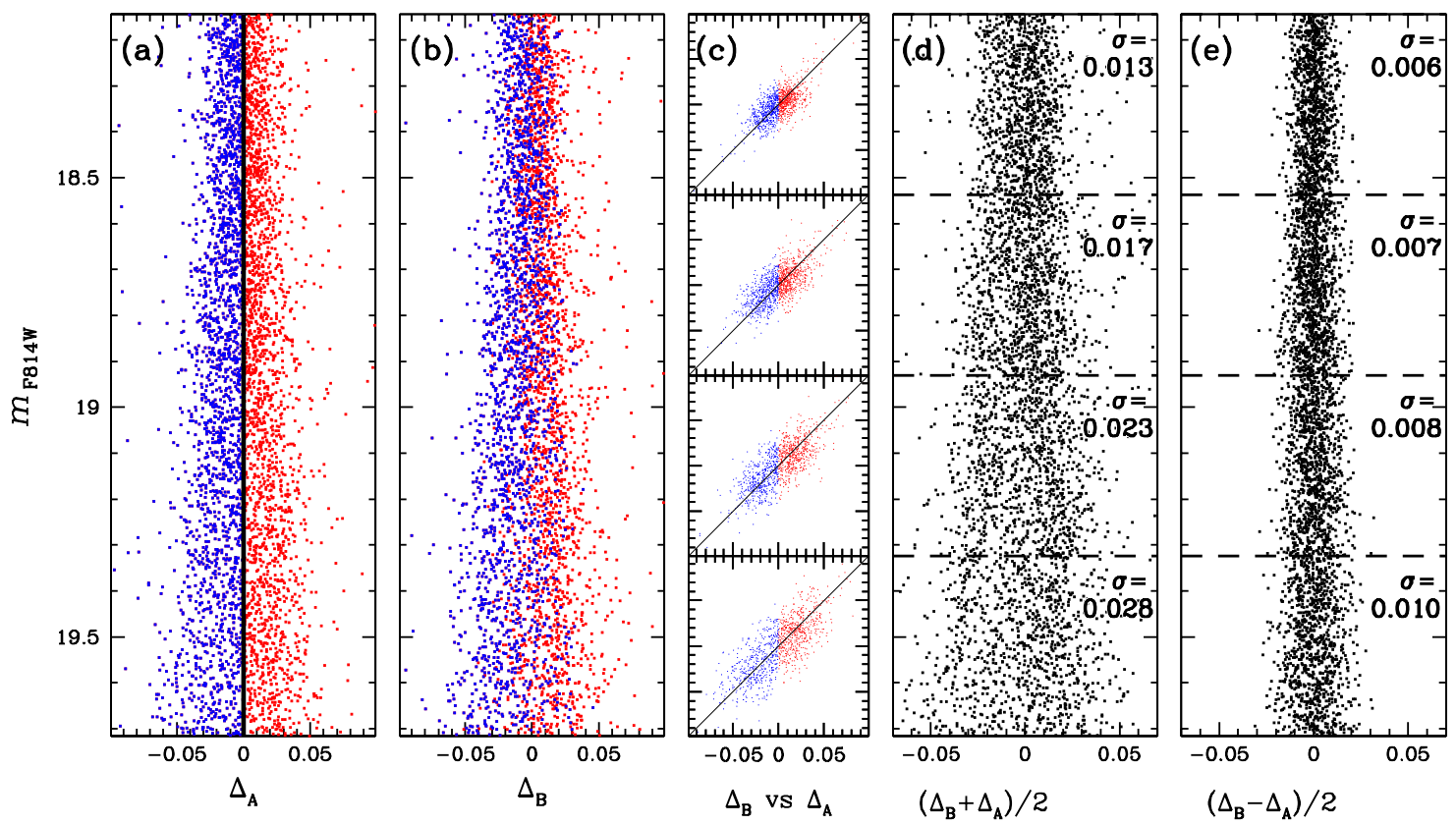

Fig. 7.- (a) Straightened MSs for the first half of the GO-10121 data. Stars with color residuals on the blue and red sides of the black vertical line are colored blue and red, respectively. (b) Straightened MSs for the second half of the data. Each star has the color that was assigned to it in the previous panel. (c) Correlations of the two color residuals, in four magnitude intervals. (d) Means of color residuals. (e) Estimates of color errors.

An additional confirmation of the presence of an intrinsically broadened MS in NGC 6752 comes from comparing each of the other data sets, on the one hand, with the entire GO-10121 data set on the other hand. Although none of the other data sets is as good as that from GO-10121, this course has the advantage that the exposures in each data set were taken at different pointings and orientations, so that a star falls on a different part of the 
detector in each of the two data sets.

In Fig. 8 we compare the main-sequence CMD from GO-10121 with CMDs from three other independent ACS data sets. Each row of panels shows our results from one data set. In the left panels we show the CMD, in two forms. Part (a) is the normal CMD; each green line is the MSRL, drawn by eye. Again, the red/blue coding of the individual stars is defined in the first straightened CMD, and maintained in the succeeding ones. Part (b) shows the straightened sequence, which is the result of subtracting from the color of each star the color of the MSRL at the magnitude of the star; this process makes the MSRL vertical. We have assigned to each star in the top diagram a red or blue color code according to whether it is on the red or blue side of the MSRL [marked by a black dividing line in the top panel of (b)], and the star keeps this color in all remaining straightened diagrams in part (b), and in all of parts (c), (d), and (f). The color displacements from the MSRL are labeled $\Delta_{i}$, where the subscript $i$ goes with the row in the figure.

This is analogous to what we showed in Fig. (7, except that there we were comparing two halves of the GO-10121 data set with each other, whereas here we start with the entire GO10121 data set, and therefore base our red-blue distinction, in panel (b) of the top row, on a reference sample that is twice as large as the one with which we began in the previous figure. In the present figure the uncertainties in each of the comparisons shown in the remaining rows are almost completely due to the second data set in the comparison, which is never as strong as that of the GO-10121 data set. What we show here, however, is independent confirmation, from images taken at other pointings and orientations, of what we found from our best data set.

The panels labeled (c) show the histograms of the distribution of color residuals $\left(\Delta_{i}\right)$ for the stars to which the red and blue symbols were assigned; they are divided into magnitude intervals, in accordance with the magnitude scales at the left edges of panels (a). In the top row the separation in panel (c) is of course perfect, because these are the colors that we defined in the preceding panel. In the other rows the separation is less sharp, but is still strongly evident. Table 2 gives the median values of the residuals $\Delta_{i}$ for the bluer stars $\left(\left\langle\Delta_{i}\right\rangle_{\mathrm{b}}\right)$, for the redder stars $\left(\left\langle\Delta_{i}\right\rangle_{\mathrm{r}}\right)$, and for the entire sample $\left(\left\langle\Delta_{i}\right\rangle_{\mathrm{ALL}}\right)$. The intrinsic color spread of the MS is confirmed by the fact that for each of the other data sets, and in all magnitude intervals within each of them, the residuals of the stars that were marked blue in the GO-10121 data set are significantly bluer than those that were marked red.

In the fourth column of panels, labeled (d), we plot $\Delta_{i}$ against $\Delta_{1}$, to further show the consistency and significance of the color spread. The solid line is the best-fitting least-squares straight line. Horizontally the separation is of course perfect, since the abscissa is $\Delta_{1}$; it is in the slope that we see the correlation. Because the different data sets have different color 
baselines, we do not expect this line to have a slope of unity, but a positive correlation indicates an intrinsic spread.

Panels (e) show the footprints of the data sets used in this analysis. The color coding is the same as in Fig. 1. The black circle indicates the region within 1 arcmin of the cluster center. Only stars in the colored areas were used in the present analysis.

Finally, panel (f), at the upper right, shows the spatial distribution of the stars of the GO-10121 data set, with the colors that they were assigned in panel (b) of the top row. No difference in radial distribution is evident. To test this better, in Figure 9 we show a Kolmogorov-Smirnov test; in random samplings from the same distribution a difference this large would occur $49 \%$ of the time.

\begin{tabular}{cccccccc}
\hline \hline CMD & mag. interval & $\left\langle\Delta_{i}\right\rangle_{\mathrm{b}}$ & $N_{\mathrm{b}}$ & $\left\langle\Delta_{i}\right\rangle_{\mathrm{r}}$ & $N_{\mathrm{r}}$ & $\left\langle\Delta_{i}\right\rangle_{\text {ALL }}$ & $N_{\text {ALL }}$ \\
\hline 1 & $18.1-18.5$ & $-0.008 \pm 0.001$ & 480 & $0.008 \pm 0.001$ & 501 & $0.000 \pm 0.001$ & 981 \\
1 & $18.5-18.9$ & $-0.011 \pm 0.001$ & 489 & $0.010 \pm 0.001$ & 521 & $0.001 \pm 0.001$ & 1010 \\
1 & $18.9-19.3$ & $-0.017 \pm 0.001$ & 465 & $0.014 \pm 0.001$ & 481 & $0.001 \pm 0.001$ & 946 \\
1 & $19.3-19.7$ & $-0.020 \pm 0.001$ & 460 & $0.018 \pm 0.001$ & 465 & $0.000 \pm 0.001$ & 925 \\
\hline 2 & $18.1-18.5$ & $-0.005 \pm 0.001$ & 478 & $0.005 \pm 0.001$ & 498 & $0.000 \pm 0.001$ & 976 \\
2 & $18.5-18.9$ & $-0.005 \pm 0.001$ & 501 & $0.006 \pm 0.001$ & 514 & $0.001 \pm 0.001$ & 1015 \\
2 & $18.9-19.3$ & $-0.006 \pm 0.001$ & 480 & $0.006 \pm 0.001$ & 478 & $0.000 \pm 0.001$ & 958 \\
2 & $19.3-19.7$ & $-0.009 \pm 0.001$ & 453 & $0.009 \pm 0.001$ & 461 & $0.002 \pm 0.001$ & 914 \\
\hline 3 & $19.4-20.0$ & $-0.006 \pm 0.001$ & 135 & $0.008 \pm 0.001$ & 138 & $0.001 \pm 0.001$ & 273 \\
3 & $20.0-20.7$ & $-0.009 \pm 0.002$ & 125 & $0.008 \pm 0.002$ & 136 & $0.000 \pm 0.001$ & 261 \\
\hline 4 & $18.1-18.9$ & $-0.006 \pm 0.001$ & 199 & $0.006 \pm 0.001$ & 207 & $0.000 \pm 0.001$ & 406 \\
4 & $18.9-19.7$ & $-0.011 \pm 0.002$ & 205 & $0.008 \pm 0.001$ & 222 & $0.000 \pm 0.001$ & 427 \\
\hline \hline
\end{tabular}

Table 2: Median color residuals for blue MS stars $\left(\left\langle\Delta_{i}\right\rangle_{\mathrm{b}}\right)$, red MS stars $\left(\left\langle\Delta_{i}\right\rangle_{\mathrm{r}}\right)$, and for the whole sample of MS stars $\left(\left\langle\Delta_{i}\right\rangle_{\mathrm{ALL}}\right)$, for each data set, in the magnitude intervals into which we have divided them. 

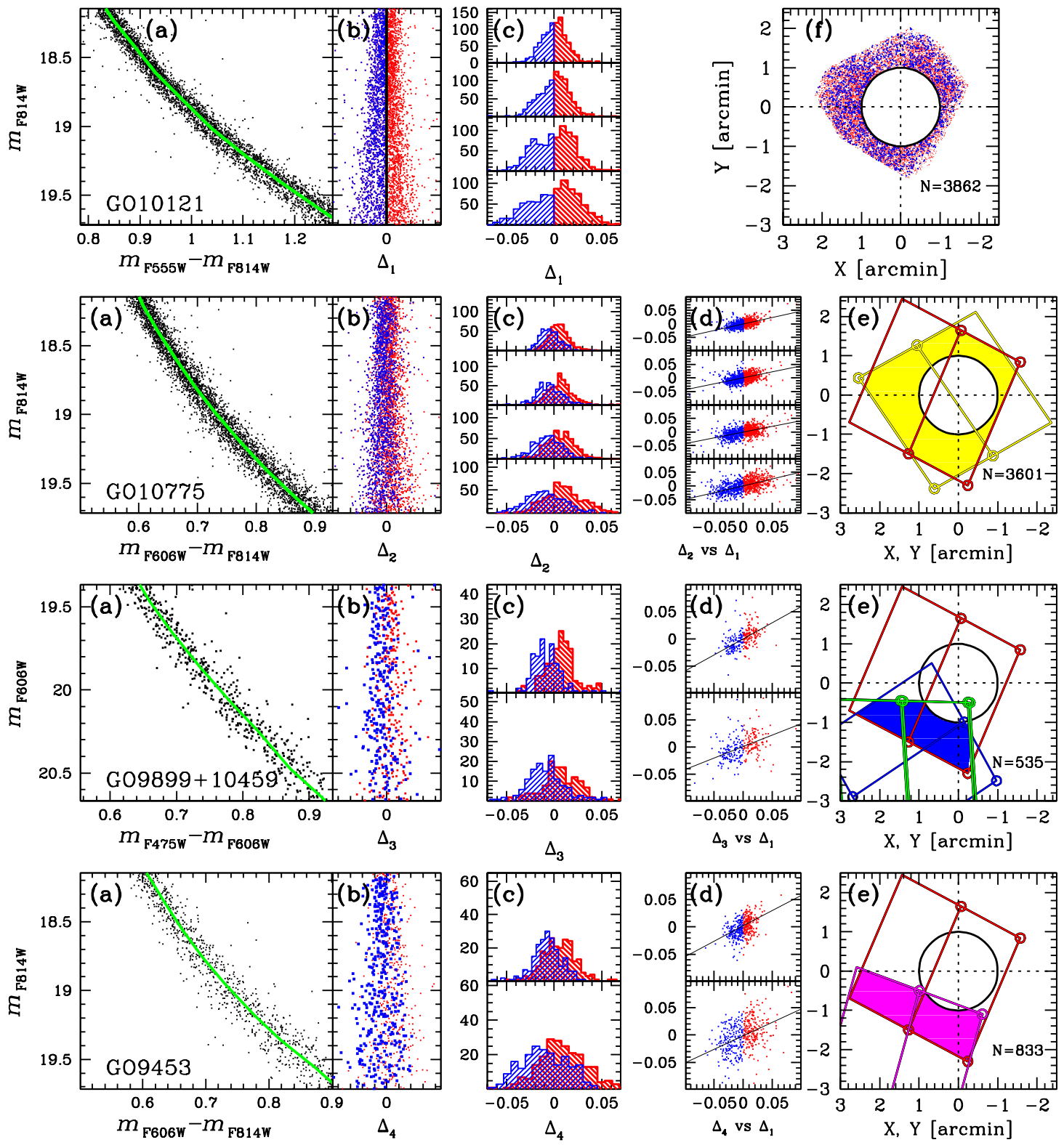

Fig. 8.- (a) CMDs of the MS stars, with MSRLs shown in green. (b) Straightened CMDs, with each star shown in the color that it was assigned in the straightened CMD of the GO10121 stars. (c) Histograms of the color residuals $\Delta_{i}$ of the stars, in magnitude intervals within each data set. Shading identifies the stars that were marked red or blue in the top row. Bi-colored cross-hatched shading is the overlap region between the blue and red histograms. (d) Correlations of color residuals of each data set with those of the GO-10121 stars; in each, the best-fitting line is superimposed. (e) Footprints of the data sets. The stars used in that row are the ones from the colored area. (f) Spatial distribution of the blue and red stars in the GO-10121 data set. 


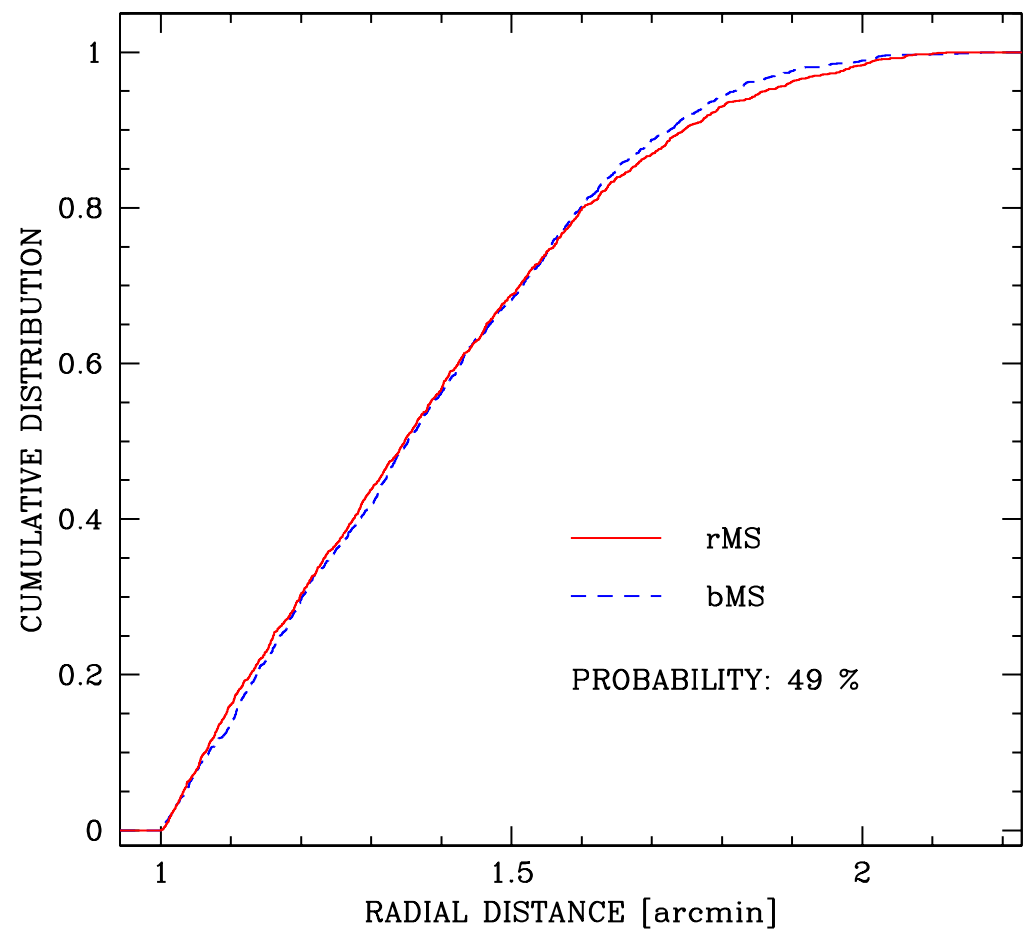

Fig. 9.- Cumulative radial distributions of the bMS and rMS stars. 


\section{The subgiant branch}

In this section we look for any evidence of multiple populations among the SGB stars of NGC 6752. The procedure that we used is illustrated in Fig. 10. In the (a) panels we show the independent CMDs from data sets 1, 2, 4 and 5 (Sect. 2), zoomed around the region of the SGB. Here we do include the central part of the cluster, because at this magnitude level crowding is not a significant photometric problem. A visual inspection at these CMDs does not reveal any significant SGB split, or even a broadening, either in magnitude or in color. There might be a few outliers below the SGB, but it is difficult to assess whether they could represent a different population, as, for instance, in NGC 1851 (Milone et al. 2008) or M22 (Marino et al. 2009), or else blends/poor photometry.

In order to provide an upper limit for the SGB spread, we drew in, by hand, the SGB ridge-lines (SGB-RLs) that are shown as red solid lines in panels (a) of Fig. 10, and computed magnitude residuals $\Delta m_{i}$ by subtracting from the magnitude of each star the magnitude of the SGB-RL at the color of the star. The residuals (note that here they are magnitude residuals, rather than color residuals, as in the earlier figures) are plotted against color in panels (b), and histograms of the $\Delta m_{i}$ distributions are shown in panels (c), while panels (d) show the footprints of the data sets, using the same color coding as in Figs. 1 and 8 , Panel (e), at the top right, shows the spatial distributions of the three magnitude subsets that we defined in panel (b) of that same row.

Once again, the CMD from the GO-10121 images has the best photometric precision, and we used it to define, in the (a) panel for that data set, two subsamples of stars brighter (bSGB) and fainter (fSGB) than the SGB-RL. We chose magenta and green for bSGB and fSGB stars respectively. In all the other diagrams we use the same color code for each star that it has in the GO-10121 data set. In the plots for each of the other data sets the median $\Delta m$ 's of the bSGB and the fSGB stars are, respectively, only marginally brighter and fainter than the median $\Delta m$ of all SGB stars. The individual values are given in Table 4 , and confirm that no significant intrinsic spread of the SGB of NGC 6752 is detectable in our data. Any intrinsic spread must be smaller than a few hundredths of a magnitude.

\begin{tabular}{ccccccc}
\hline \hline CMD & $\left\langle\Delta m_{i}\right\rangle_{\mathrm{bSGB}}$ & $N_{\mathrm{bSGB}}$ & $\left\langle\Delta m_{i}\right\rangle_{\mathrm{fSGB}}$ & $N_{\mathrm{fSGB}}$ & $\left\langle\Delta m_{i}\right\rangle_{\mathrm{ALL}}$ & $N_{\mathrm{ALL}}$ \\
\hline 1 & $0.018 \pm 0.001$ & 249 & $-0.011 \pm 0.001$ & 273 & $0.001 \pm 0.001$ & 522 \\
2 & $0.010 \pm 0.002$ & 197 & $-0.004 \pm 0.002$ & 233 & $0.002 \pm 0.001$ & 430 \\
3 & $0.012 \pm 0.006$ & 28 & $-0.014 \pm 0.005$ & 30 & $-0.003 \pm 0.005$ & 58 \\
5 & $0.004 \pm 0.006$ & 21 & $0.003 \pm 0.006$ & 23 & $0.004 \pm 0.004$ & 44 \\
\hline
\end{tabular}

Table 3: Average magnitude residuals for bright SGB stars $\left(\left\langle\Delta m_{i}\right\rangle_{\mathrm{bSGB}}\right)$, faint SGB stars $\left(\left\langle\Delta m_{i}\right\rangle_{\mathrm{fSGB}}\right)$, and for all the SGB stars $\left(\left\langle\Delta_{i}\right\rangle_{\mathrm{ALL}}\right)$ in data sets $1,2,3$, and 5.

Note that this result really comes only from the GO-10121 and GO-10775 data sets. 
The other data sets have too few stars to contribute anything significant; we show them only for completeness, and to verify that they do not show anything that would contradict our conclusion.

As for the radial distributions, Figure 11] compares them for bSGB and fSGB stars. Although the difference would appear to be large, the Kolmogorov-Smirnov statistic indicates that in random samplings from the same distribution a difference this large would occur 4 $\%$ of the time, suggesting that a real difference is possible, but far from conclusive. 

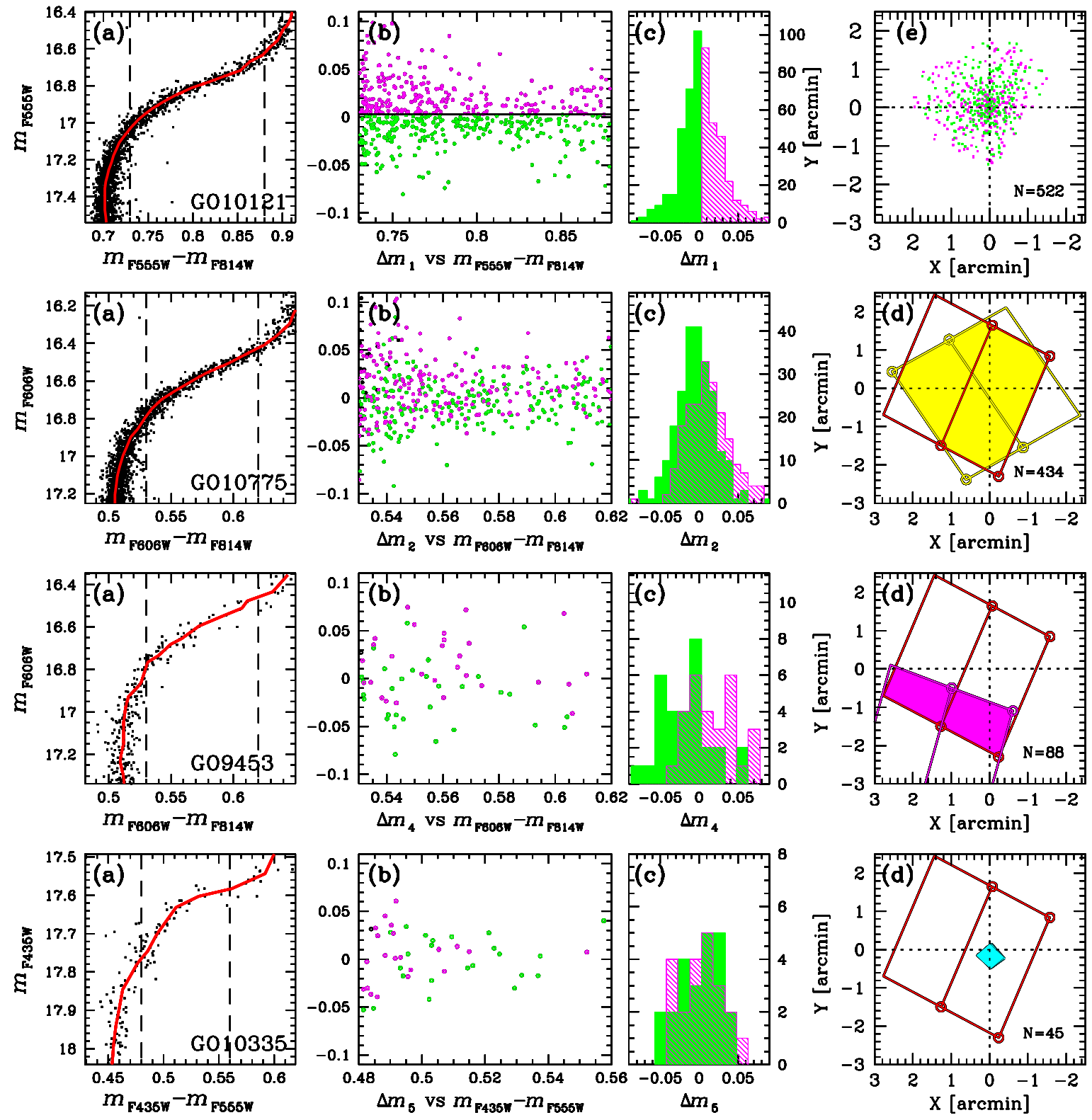

Fig. 10.- (a) Zoom of the CMDs around the SGB. (b) Straightened SGBs, with the samples of fSGB, and bSGB stars (faint, and bright SGB) colored green, and magenta, respectively. In the top row, the stars are from the GO-10121 CMD, in the other rows, from the colored area in panels (d). (c) Distributions of the magnitude residuals $\Delta m_{i}$. (d) Footprints of the data sets. The stars used in this analysis come from the colored area. (e) (at upper right): Spatial distributions of the fSGB and bSGB stars (in green, and magenta, respectively). 


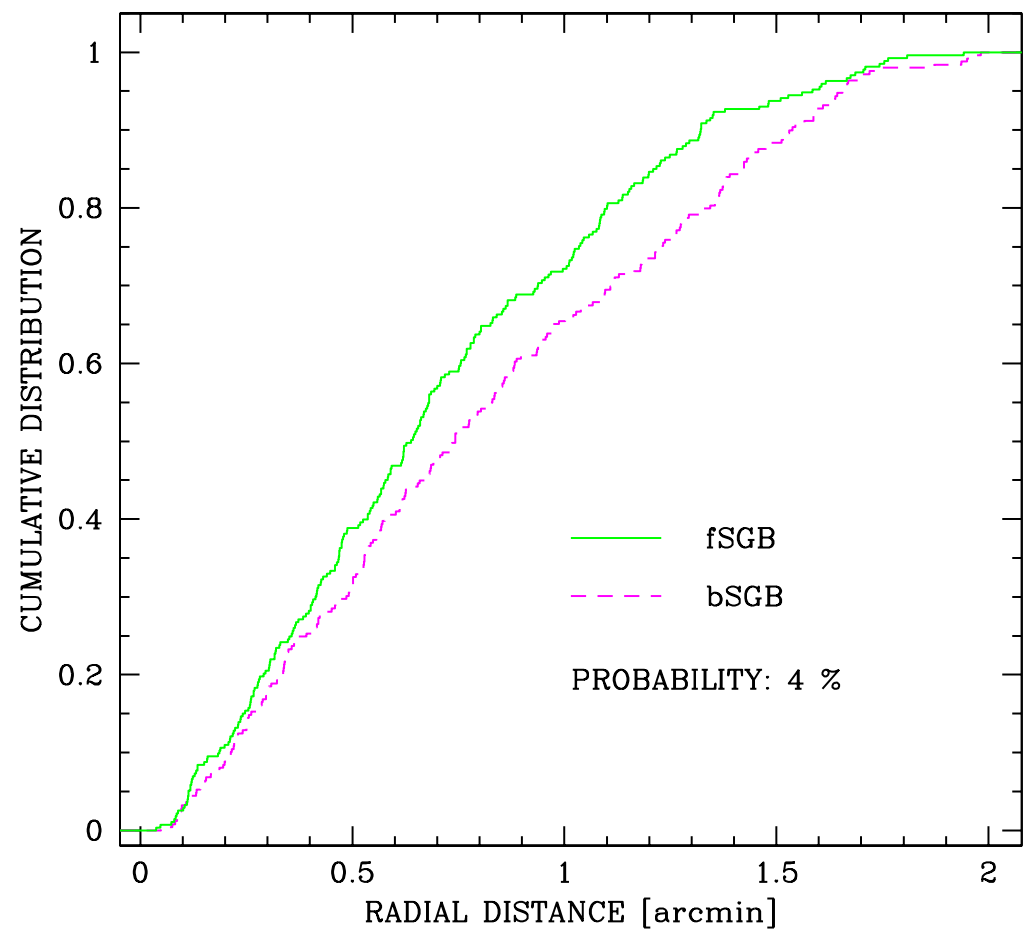

Fig. 11. - Cumulative radial distributions of the bSGB and fSGB stars. 


\section{Na and $\mathrm{O}$ abundances along the broad red-giant branch}

Photometric evidence for a broadening of the RGB of NGC 6752 was found by Grundahl et al. (2002) and Yong et al. (2008). Both papers found that the Strömgren photometric index $c_{1}$ correlates with the nitrogen abundance, and concluded that the observed scatter in this index is due to variations in $\mathrm{NH}$ band strength, in stars both brighter and fainter than the RGB bump.

A broadened RGB is also visible in a $U$ vs. $U-B$ CMD of NGC 6752, derived from ground-based photometry by Momany et al. (2002) with ESO's Wide Field Imager, and shown in Fig. 12, The broadening extends from the base of the RGB to the RGB tip, and looks extremely similar to the broadening of the RGB observed in M4, and associated by Marino et al. (2008) with the presence of two stellar populations with different ratios in their $[\mathrm{Na} / \mathrm{Fe}]$ and $[\mathrm{O} / \mathrm{Fe}]$ abundances, and different $\mathrm{CN}$ band strengths. The two groups of stars studied by Marino et al. (2008) in M4 lie in two different regions of the RGB. One group, which consists of Na-rich, CN-strong stars, occupies a narrow sequence on the red side of the RGB, while the other group, made up of Na-poor and CN-weak stars, has a broader spread on the blue side of the RGB. (See Marino et al. 2008 for more details on the abundances of RGB stars in M4.)

The Na-O anticorrelation in NGC 6752 has been extensively studied by Gratton et al. (2001), and Carretta et al. (2005, 2007, 2009). In particular, both Gratton et al. (2001) and the 2005 Carretta et al. paper also found the Na-O anti-correlation in unevolved and barely evolved stars. Carretta et al. (2007, 2009) present an analysis of the largest sample of globular cluster RGB stars available in the literature. In Fig. 12 we identify stars from Carretta et al. (2007) in our $U$ vs. $U-B$ CMD, by using the same symbols as in the inset, which shows the Na-O anticorrelation that they found. Here, following the criteria defined in Carretta et al. (2009), we isolate two subsamples: $i$ ) the primordial population, located in the Na-poor/O-rich region (marked with blue symbols), and $i i$ ) their extreme and intermediate components, which are Na-rich/O-poor. (We have lumped these two groups together as red symbols).

The same two groups of stars are located in different regions of the RGB in the $U$ vs. $U-B$ CMD, in close analogy with the results by Marino et al. (2008) for M4. Most of the Na-poor (primordial) stars are distributed on a bluer and broader sequence around the RGB, while the Na-rich stars tend to be distributed on a narrower sequence on the red part of the RGB. This stellar distribution continues well below the HB level. The bimodal color distribution also extends well below the RGB-bump, suggesting that it is not a consequence of the mixing of the stellar interiors. The MS of NGC 6752 is intrinsically broadened, and possibly split. Also, the Na-O anti-correlation is more extended in NGC 6752 than in M4. 


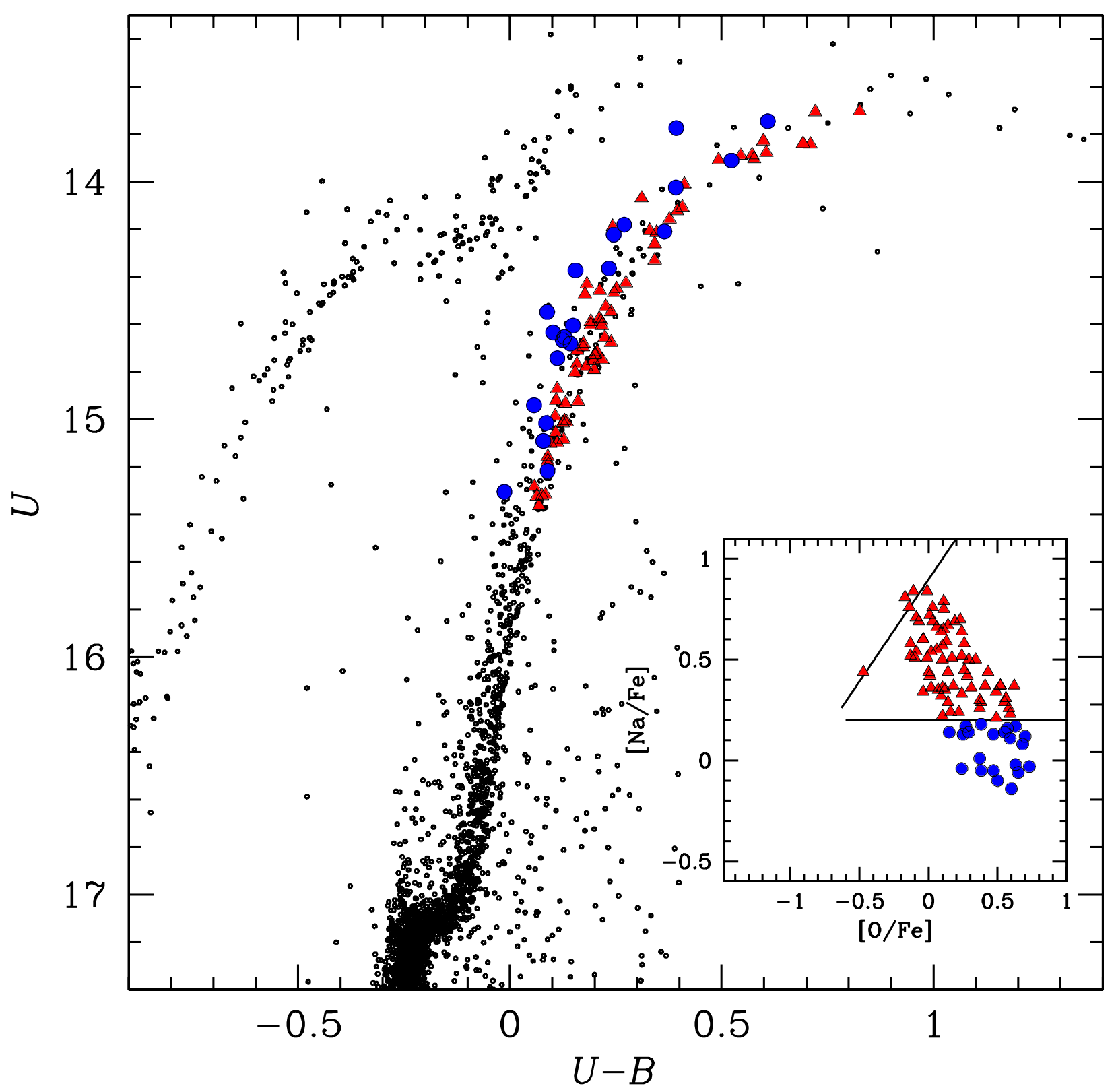

Fig. 12.- $U$ vs. $(U-B)$ CMD from Wide Field Imager photometry, as obtained by Momany et al. (2002). The Na-O anti-correlation for the stars measured by Carretta et al. (2007) is shown in the inset, where the solid lines delimit the primordial, intermediate, and extreme populations, as defined in Carretta et al. The stars of the primordial population are represented by blue circles, while red triangles are the intermediate and extreme groups. 


\section{Discussion}

The CMDs presented in this paper demonstrate that NGC 6752 hosts a stellar population that has complexities similar to those identified in other GCs investigated so far (see Piotto 2009 for a recent review). This cluster exhibits a broadened MS, with some evidence of a split, while the SGB does not show any significant vertical spread, even at the level of a few hundredths of a magnitude. The MS broadening is intrinsic, and not due to binaries or photometric errors. The RGB is significantly broadened both in the Strömgren $c_{1}$ index (Yong et al. 2008) and in the $U-B$ color. Stars that are O-poor and Na-rich mainly populate a sequence on the red RGB portion of the $U$ vs. $U-B$ CMD, while O-rich/Na-poor stars are preferentially located on the blue side of the RGB, with a broader color distribution.

It is interesting to look for similarities between NGC 6752 and the other clusters with photometric evidence of multiple stellar populations. The only two other clusters that are known to have multiple MSs (i.e., with clearly separated branches, rather than just a broadening) are $\omega$ Cen and NGC 2808. Starting from the CMD morphologies and the information we have on their chemical abundance patterns, and by analogy with the current interpretation of the multiple-MS phenomenon, we can hypothesize that the stars on the blue side of the MS of NGC 6752 could belong to a second stellar generation, born from material polluted by a previous population of stars, and He-enriched. We note that a large amount of He has also been invoked to interpret the blue HB tails which are present in many clusters (D'Antona et al. 2005). Interestingly, $\omega$ Centauri, NGC 2808, M54, and NGC 6752 all have such extended blue HBs.

In an increasing number of clusters, including NGC 1851, M22, and NGC 6388, the presence of multiple stellar populations is inferred from the split SGB, but no evidence of a split or spread-out MS has been found so far in these objects. (Some evidence of a split in the SGB has been found in 47 Tuc too, by Anderson et al. 2009, though that case lacks the clear separation shown in the other clusters that we have mentioned.) The split SGB of NGC 1851 has been interpreted as coming from two stellar populations with large differences in the total $\mathrm{C}+\mathrm{N}+\mathrm{O}$ abundance (Cassisi et al. 2008, Ventura et al. 2009). Indeed, large variations in the total $\mathrm{C}+\mathrm{N}+\mathrm{O}$ content among four bright RGB stars of NGC 1851 have been reported by Yong et al. (2009). In NGC 1851 and M22 the two SGBs may be related to the two groups of stars with a large difference in the abundances of $s$-process elements $(\sim 0.4 \mathrm{dex})$, identified by Yong \& Grundahl (2008) and Marino et al. (2009), respectively.

In NGC 6752 the overall sum $\mathrm{C}+\mathrm{N}+\mathrm{O}$ is almost constant (Carretta et al. 2007). This cluster presents a large star-to-star abundance variation in N (1.95 dex, Yong et al. 2008), and the amplitude of the abundance variations for $s$-process elements is small $(<0.2 \mathrm{dex}$, Yong et al. 2008). 
If the polluting matter is identified with the envelopes of asymptotic giant branch stars (Ventura et al. 2001, 2002), these AGB stars should be very massive in order to experience the second dredge-up, which brings a large amount of fresh He to the envelope, and the hot bottom burning process, which converts C into N. Such massive AGB stars should not live long enough, however, for a sufficient number of them to experience a third dredge-up and significantly increase the total $\mathrm{C}+\mathrm{N}+\mathrm{O}$ abundance in the envelope (Renzini 2008). As an alternative, polluted material could come from the envelopes of fast-rotating stars during the H-burning phase; in this case too, we expect a He-enriched second generation without any $\mathrm{C}+\mathrm{N}+\mathrm{O}$ enhancement at all (Decressin et al. 2007).

At this stage, observational insight into such processes could come from a detailed spectroscopic analysis of stars on the blue and red sides of the MS and of the RGB. In addition, the new WFC3 camera, which is sensitive to both the UV and IR spectral regions, should give a wide color baseline, offering a unique opportunity to better distinguish the signatures of multiple stellar populations in the CMD. Such a study would allow not only a better characterization of the complex case of NGC 6752, but also a more consistent interpretation of the observations of multiple stellar populations in GCs in general.

Acknowledgements We are very thankful to Raffaele Gratton and Eugenio Carretta for generously sharing with us their spectroscopic catalogs of $\mathrm{Na}$ and $\mathrm{O}$ abundances in stars, in electronic format, and for the many fruitful discussions. We also thank Antonio Sollima for useful discussion. APM, AFM, and GP acknowledge support by PRIN2007 (prot. n. 20075TP5K9), and by ASI under the program ASI-INAF I/016/07/0. IRK and JA acknowledge support by STScI grants GO9899 GO-10922, and GO-11233. LM acknowledges support by the STScI 2009 Space Astronomy Summer Program.

\section{REFERENCES}

Anderson, J., \& King, I. R. 1999, PASP, 111, 1095

Anderson, J., \& King, I. R. 2000, PASP, 112, 1360

Anderson, J., \& King, I. R. 2003, PASP, 115, 113

Anderson, J., \& King, I. R. 2004, ACS ISR 2004-15

Anderson, J., \& King, I. R. 2006, ACS ISR 2006-01

Anderson, J., et al. 2008, AJ, 135, 2055 
Anderson, J., Piotto, G., King, I. R., Bedin, L. R., \& Guhathakurta, P. 2009, ApJ, 697, L62

Bedin, L. R., Anderson, J., King, I. R., \& Piotto, G. 2001, ApJ, 560, L75

Bedin, L. R., Piotto, G., Anderson, J., Cassisi, S., King, I. R., Momany, Y., \& Carraro, G. 2004, ApJ, 605, L125

Bedin, L. R., Cassisi, S., Castelli, F., Piotto, G., Anderson, J., Salaris, M., Momany, Y., \& Pietrinferni, A. 2005, MNRAS, 357, 1038

Carretta, E., Gratton, R. G., Lucatello, S., Bragaglia, A., \& Bonifacio, P. 2005, A\&A, 433, 597

Carretta, E., Bragaglia, A., Gratton, R. G., Lucatello, S., \& Momany, Y. 2007, A\&A, 464, 927

Carretta, E. et al. 2009, A\&A, 505, 117

Cassisi, S., Salaris, M., Pietrinferni, A., Piotto, G., Milone, A. P., Bedin, L. R., \& Anderson, J. 2008, ApJ, 672, L115

D’Antona, F., Bellazini, M., Caloi, V., Fusi Pecci, F., Galleti. S., \& Rood, R. T. 2005, ApJ, 631,868

Decressin, T., Meynet, G., Charbonnel, C., Prantzos, N., \& Ekström, S. 2007, A\&A, 464, 1029

Girardi, L., Groenewegen, M. A. T., Hatziminaoglou, E., \& da Costa, L. 2005, A\&A 436, 895

Gratton, R. G., et al. 2001, A\&A, 369, 87

Gratton, R. G., Sneden, C., \& Carretta, E. 2004, ARA\&A, 42, 385

Grundahl, F., Briley, M., Nissen, P. E., \& and Feltzing, S. 2002, A\&A, 385, L14

Harris, W. E. 1996, AJ, 112, 1487 (February 2003 update)

Kroupa, P. 2002, in ASP Conf. Ser. 285, Modes of Star Formation and the Origin of Field Populations, eds. E. K. Grebel \& W. Brandner (San Francisco: ASP), p. 86

Landolt, A. U. 1992, AJ, 104, 372

Lee, Y.-W., Joo, J.-M., Sohn, Y.-J., Rey, S.-C., Lee, H.-C., \& Walker, A. R. 1999, Nature, 402,55 
Mackey, A. D., Broby Nielsen, P., Ferguson, M. N., \& Richardson, J. C. 2008, ApJ, 681, L17

Marino, A. F., Villanova, S., Piotto, G., Milone, A. P., Momany, Y., Bedin, L. R., \& Medling, A. M. 2008, A\&A, 490, 625

Marino, A. F., Milone, A. P., Piotto, G., Villanova, S., Bedin, L. R., \& Renzini, A. 2009, A\&A, 505, 1099

Milone, A. P., et al. 2008, ApJ, 673, 241

Milone, A. P., Piotto, G., Bedin, L. R. \& Sarajedini, A. 2008b, in XXI Century Challenges for Stellar Evolution, Memorie della Societa Astronomica Italiana, vol. 79/2, eds: S. Cassisi, M. Salaris (arXiv:0801.3177)

Milone, A. P., Bedin, L. R., Piotto, G., \& Anderson, J. 2009, A\&A, 497, 755

Momany, Y., Piotto, G., Recio-Blanco, A., Bedin, L., R., Cassisi, S., \& Bono, G. 2002 ApJ, 576, L65

Moretti, A., et al. 2008, A\&A, 493, 539

Norris, J. E. 2004, ApJ, 612, L25

Pancino, E., Ferraro, F. R., Bellazzini, M., Piotto, G., \& Zoccali, M. 2000, ApJ, 534, L83

Piotto, G., et al. 2005, ApJ, 621, 777

Piotto, G., Bedin, L. R., Anderson, J., King, I. R., Cassisi, S., Milone, A. P., Villanova, S., Pietrinferni, A., \& Renzini, A. 2007, ApJ, 661, L53

Piotto, G., 2009, in IAU Symposium No. 258, the Ages of Stars, eds. E. E. Mamajek, D. R. Soderblom, \& R. F. G. Wyse (Cambridge: Cambridge Univ. Press), p. 233 (arXiv:0902.1422)

Renzini, A., \& Buzzoni, A. 1986, in Spectral Evolution of Galaxies, eds. C. Chiosi \& A. Renzini (Dordrecht: Reidel), ASSL, 122, p. 195

Renzini, A. 2008, MNRAS, 391, 354

Rubenstein, E. P., \& Bailyn, C. D. 1997, ApJ, 474, 701 [RB97]

Sirianni, M., et al. 2005, PASP, 117, 1049

Sollima, A., Beccari, G., Ferraro, F. R., Fusi Pecci, F., \& Sarajedini, A. 2007, MNRAS, 380, 781 
Stetson, P. B. 1994, PASP, 106, 250

Valdes, F. G. 1998, in ASP Conf. Ser. 145, Astronomical Data Analysis Software and Systems VII, eds. R. Albrecht, R. N. Hook, \& H. A. Bushouse (San Francisco: ASP), p. 53

Ventura, P., D’Antona, F., Mazzitelli, I., \& Gratton, R. 2001, ApJ, 550, 65

Ventura, P., D’Antona, F., \& Mazzitelli, I. 2002, A\&A 393, 215

Ventura, P., Caloi, V., D’Antona, F., Ferguson, J., Milone, A., \& Piotto, G. 2009, MNRAS, in press (arXiv:0907.1765)

Villanova, S., et al. 2007, ApJ, 663, 296

Yong, D., Grundahl, F., Johnson, J. A., \& Asplund, M. 2008, ApJ, 684, 1159

Yong, D., \& Grundahl, F. 2008, ApJ, 672, L39

Yong, D., Grundahl, D’Antona, F., Karakas, A. I., Lattanzio, J. C., \& Norris, J. E. 2009, ApJ, 695, L72 